\title{
DAR Y MANTENER VIDA EN LAS REPRESENTACIONES \\ FIGURATIVAS FEMENINAS DE VALDIVIA (ECUADOR): UN \\ ANÁLISIS CRÍTICO ${ }^{1}$
}

\author{
María Dolores Guerrero Perales²
}

\begin{abstract}
Dar y mantener vida en las representaciones figurativas femeninas de Valdivia (Ecuador): un análisis crítico

Resumen: La mayor parte de las investigaciones que tratan las Figuras de Valdivia, conocidas y denominadas comúnmente como "Venus de Valdivia", lo han hecho omitiendo y marginando elementos tan significativos como son la tarea producir y mantener vida. Es por ello, que desde una Arqueología Feminista, se pretende estudiar aquellas representaciones en las que las mujeres aparecen desempeñando estos trabajos, los cuales, consideramos que son actividades económicas fundamentales en las sociedades del pasado. Dichas labores se manifiestan mediante la representación de mujeres embarazadas, dando a luz o sujetando una criatura en brazos.

Palabras clave: Representaciones, mujeres, Valdivia, feminismo, arqueología, social, Ecuador.

Giving and Maintaining Life in the Female Figure Representations of Valdivia (Ecuador): A Critical Analysis

Abstract: Most of the investigations about Valdivia figures, known and commonly called "Venus of Valdivia" have been done by the omission and marginalization of elements that are so significative like the activities of produce and keep life. For this reason, from a Social Archaeology, we pretend to study these representations where women appear doing this works, which we consider that are essential economic activities for the societies of the past. These jobs, are manifested by the representations of women giving birth, pregnant or holding a child in arms. Keywords: Representations, women, Valdivia, feminism, arqueology, social, Ecuador.
\end{abstract}

\section{Introducción}

El estudio de las figuras de Valdivia (Ecuador), en general, se ha venido abordando desde dos perspectivas principales: desde el ámbito de la Historia del Arte con un análisis basado en los estilos y caracterizado por una mirada estética, y desde un punto de vista arqueológico.

${ }^{1}$ Fecha de recepción: 23/11/2016.

Fecha de aceptación: 15/12/2016. 2 Investigadora en formación del Departamento de Prehistoria. Miembro del grupo de investigación ACAIA, Universitat Autònoma de Barcelona; $\square$ mdgperales@gmail.com. 
Si nos centramos en esta última disciplina, podemos observar que la mayor parte de las investigaciones han basado su discurso en el establecimiento de diversas tipologías ${ }^{1}$ de las figuras (Capua 2002; Hill 1972; López 1996; García 2006) y en llevar a cabo hipótesis que en su mayoría, otorgan un carácter ceremonial a las mismas. En general, se tiende a observar una tendencia por parte de la mayoría de la comunidad científica a darle un sentido ritual a aquellos elementos que se escapan de nuestro conocimiento. Nos referimos a un tipo de concepción que intenta rescatar el pasado o las "raíces", tomando como base una determinada estética, propia de la disciplina de las Bellas Artes. Para ello se empleará el arte primitivo, la etnografía, etc. como elementos de búsqueda de unos orígenes étnico-nacionales o culturales, que llevan consigo un arraigo estético y sexista ${ }^{2}$ (Castro y Escoriza 2009: 40).

A través de nuestro análisis, pretendemos alejarnos de este tipo de perspectivas y acercarnos al estudio de las figuras de Valdivia como materialidad social, producto de un trabajo específico, y que, a su vez, aparecen representando otro trabajo: el dar y el mantener vida. De esta manera, pretendemos realizar una Sociología desde la Arqueología. El marco teórico del que partimos se conforma por: la Teoría de las Representaciones Figurativas y la Teoría de la Producción de la Vida Social. Respecto a la primera, ésta consiste en el estudio de las representaciones figurativas contempladas como cualquier otro objeto material. Así mismo, debe encontrar su base en el análisis de las condiciones materiales en las que fue producido, las cuales abarca también su uso social (Escoriza y Sanahuja 2002). Las representaciones figurativas, son gestionadas en situaciones de relación, fruto de las prácticas sociales que realizan mujeres y hombres, por este motivo, nos darán información en los contextos arqueológicos en los que estén implicados (Escoriza 2002: 28). Es necesario para ello, sexuar las figuras, de manera que son los órganos sexuales que aparezcan representados en las mismas los que nos aporten

\footnotetext{
1 Atendiendo fundamentalmente a determinadas características representadas en las mismas (representación/tamaño de los atributos sexuales, tipo de tocado, posición de las manos, etc.) y en relación al yacimiento en el que fueron halladas.

${ }^{2}$ Este tipo de planteamientos encontraron su auge con la llegada del Postmodernismo, donde el conocimiento de la realidad y las condiciones materiales de los hombres y mujeres del pasado, tenía un lugar secundario (Castro y Escoriza 2009: 40).
} 
la información pertinente. Pero cuando estos órganos no aparecen, tenemos que basarnos en otro tipo de elementos que se muestren relacionados con cada sexo (Escoriza 2002).

Sin embargo, es la Teoría de la Producción de la Vida Social la que tiene un mayor peso en el presente estudio. Esta teoría, afirma que los objetos y los sujetos sociales (mujeres y hombres) se suman a la producción de la vida social, manifestándose como fuerza de trabajo, medios de trabajo y productos. Las mujeres y los hombres, se contemplan como condiciones independientes, de manera que la producción de sujetos sociales conforma un elemento determinante y fundamental en la vida social. Esta tarea solo recae y depende del colectivo femenino, el cual creará a los futuros sujetos sociales que realizarán y serán destinatarios/as del trabajo humano. Actividades como la Producción de Mantenimiento, de la cual hablaremos posteriormente, sería considerada igualmente como una actividad económica fundamental (Castro et al 2005; Sanahuja, 2002).

\section{Las Figuras de Valdivia}

En primer lugar, es indispensable conocer qué son las Figuras de Valdivia (comúnmente denominadas "venus ${ }^{3}$ “), enmarcarlas en un contexto cronológico y espacial; y en segundo lugar las analizaremos en base a la Teoría de las Representaciones Figurativas (Escoriza y Sanahuja 2002; Sanahuja, 2002) y la Teoría de la Producción de la Vida Social (Castro et al 2005; Sanahuja 2002). Así mismo, nos centraremos en aquellas figuras que representen las actividades de dar y mantener vida.

La sociedad que produjo estas figuras es denominada comúnmente como Sociedad de Valdivia. Ésta ocupó el territorio que actualmente comprenden las provincias de Guayas, Isla Puná, Los Ríos, Manabí y El Oro, es decir, las tierras bajas de la costa oeste

\footnotetext{
${ }^{3}$ Comúnmente, a estas figurillas se las ha denominado "Venus", término que también se ha relacionado con las figurillas femeninas del Paleolítico Superior (30.000 - 10.000 cal ANE). Con este concepto se ha pretendido hacer un paralelismo con las diosas romanas del amor y de la belleza. Pero estas figuras prehistóricas, poco tienen que ver con esta idea, puesto que el nombre de "Venus" engloba un trasfondo de ideal erótico y canon de belleza, escondido bajo la perspectiva patriarcal actual (Castro y Escoriza 2011: 107).
} 
de Ecuador. Se trata de una ocupación bastante larga, abarcando el llamado Periodo Formativo Temprano, concretamente desde el 4400 al 1450 cal ANE. Este largo periodo fue dividido en 8 fases con dataciones radiocarbónicas en base a una secuencia de la cerámica (Hill 1975).

Respecto al asentamiento, éste estaba organizado en torno a una plaza central donde se localizaban las unidades interpretadas comúnmente como cívico-ceremoniales. Se calcula que durante el periodo de mayor densidad demográfica (2425 - 2250 cal ANE), pudieron habitar entre 1500 y 3000 personas $^{4}$ (Baumann 1985: 79). Tenían una economía basada en la horticultura con el cultivo de productos como achira, lerén, ají o yuca, aprovechando la fertilidad que aportaban los ríos Verde y Zapotal. Esta dieta, era complementada por la recolección y pesca de productos marinos como pescado o moluscos (Lathrap y Marcos 1975; Zevallos Menéndez, 1971; en Marcos 2012: 17). Cabe destacar la importancia de esta sociedad, por ser considerada una de las primeras en llevar a cabo asentamientos urbanos con una clara estructura que podríamos considerar como ciudad $^{5}$ y uno de los posibles orígenes de la producción cerámica en América del Sur (Barroso 2014).

Respecto a la organización social de estas poblaciones, muchas hipótesis ponen de manifiesto el posible comienzo de las disimetrías sociales, con argumentos basados en la división social del trabajo existente entre asentamientos principales y secundarios. Esta división se fundamenta en las labores de distribución que llevarían a cabo los asentamientos principales sobre los productos obtenidos y transformados en los asentamientos secundarios. Un nuevo elemento primordial a investigar sería, si además de una división social pudo darse o no algún tipo de división sexual del trabajo dentro de

\footnotetext{
${ }^{4}$ Destaca la gran cantidad de material encontrado en algunas de las viviendas. Por ejemplo, en una capa de unos 20 centímetros, se hallaron centenares de vasijas cerámicas y figuritas ( 2300 - 2200 cal ANE).

${ }^{5}$ Consideramos una ciudad como una comunidad de asentamiento base sedentario, la cual no produce los alimentos necesarios, es mayor que las comunidades de carácter rural que producen la comida, y cuenta con lugares de encuentro. En la Prehistoria, en las sociedades ágrafas, podemos encontrar casos en los que no se dan las relaciones de ciudad con especialización, Estado y explotación. La manifestación material de estas comunidades es el urbanismo. En éstos, se realizan prácticas sociales, es decir, hay lugares sociales donde se llevan a cabo actividades en las que se ven involucrados mujeres, hombres y objetos materiales, donde se realizan trabajos (económicos o político-ideológicos), donde se utilizan, consumen, disfrutan y sufren productos, y donde se establecen relaciones sociales. Para los grupos domésticos, sus espacios son las unidades domésticas, donde se llevan a cabo la prácticas sociales (Castro et al 2003: 1-2).
} 
estos asentamientos, ya que de esta manera podríamos acercarnos a la existencia de algún tipo de explotación de un colectivo sexual sobre otro.

Otra cuestión que defienden algunos/as autores/as, es la posible existencia de un matriarcado o sociedad matrilineal, partiendo de dos premisas fundamentales: la primera se basa en el estudio etnográfico realizado sobre otras comunidades ecuatorianas con una economía similar a la de Valdivia, en las cuales, la propiedad de la tierra y el linaje pasa de madres a hijas. La segunda, centra su atención en un característico enterramiento de Real Alto. En éste, se hallaron el cuerpo de una mujer y ocho hombres, siendo ella la que ocupaba el lugar principal de la sepultura, mientras que uno de los cuerpos de éstos, apareció desmembrado. Para algunos/as investigadores/as, pueden ser reflejo de un sacrificio para la mujer (Ayala y Fernández 1995: 20; Barroso 2009: 9). Sin embargo, para hablar de una sociedad matriarcal, tendríamos que poder observar en el registro arqueológico, un dominio, violencia, coerción o explotación del colectivo femenino sobre el colectivo masculino, y no concebirla como tal cuando vemos que una mujer adquiere cierto rango social elevado o tiene poder. Además, esta coerción no se daría solo en el interior del grupo de parentesco, sino que se extendería a toda la esfera pública (Sanahuja, 2002).

En lo que a las figuras de Valdivia se refiere, éstas fueron producidas entre los años 4400 y 1500 cal ANE. Se trata de representaciones de mujeres realizadas en piedra durante las primeras fases, y en arcilla posteriormente. El sexo que se ha pretendido manifestar, se distingue perfectamente gracias a la representación de los senos y la vulva. Respecto a las figuras de piedra, el paso de piezas aplanadas de piedra como materia prima para su elaboración, a guijarros elipsoidales, permitió un aumento de la capacidad para esculpir con mayor destreza y complejidad los órganos sexuales. Aún así, cierta parte de la comunidad científica, sostiene que algunas de estas figuras en piedra se podrían sexuar como masculinas ${ }^{6}$, a pesar de que no se haya representado ningún pene en éstas (Capua 2002: 144). De momento, no se han hallado figuras en estado de gestación, dando a luz o con alguna criatura en los brazos para estas primeras fases.

\footnotetext{
6Éstas se han asociado comúnmente con una tipología de figuras denominadas como "Palmar inciso".
} 
Fue a partir de la Fase II (3300 - 2800 cal ANE), cuando se comenzaron a realizar las figuras en cerámica. El modo de manufactura consistía en una primera unión de dos cordeles de arcilla para luego, una vez unidos (a excepción de la parte correspondiente a las piernas), ir dando forma al cuerpo femenino. Se daba forma redondeada a lo que conformaría la cabeza, el pecho y las caderas, y se estrechaba la figura a la altura del cuello, el talle y el final de las piernas, conformadas por los dos cordeles. La mayoría presentaban un acabado de engobe rojo y una serie de detalles elaborados mediante incisiones para representar el pelo, los brazos o los rasgos de la cara (López 1996: 168). En general, todas las figuras han aparecido en gran diversidad de contextos, es decir, tanto en unidades domésticas como en los llamados centros cívico-ceremoniales ${ }^{7} \mathrm{o}$ en enterramientos, como el Osario de Real Alto mencionado anteriormente.

Una de las principales hipótesis, que a día de hoy ha sido bastante aceptada por la comunidad científica, es la del papel que pudieron tener estas figuras como elemento protagonista en un "Ritual de Pubertad". Éstas serían reflejo de una edad o fase biológica determinada de las mujeres dependiendo del tipo de rapado o atributos sexuales que se representara en las mismas. Dicho ritual, consistiría en lo que se ha observado en otros pueblos de las cuencas de los grandes ríos de América del Sur, donde se celebra una ceremonia de pubertad femenina, basado en el arranque del pelo de las jóvenes (Capua, 2002). Sin embargo, no se ha encontrado ningún indicio o evidencia en el registro arqueológico que confirme tal interpretación.

Las figuras en posición de gestar, dar y mantener vida, se comenzaron a realizar en mayor medida durante las fases VII y VIII (1950 - 1450 cal ANE). A pesar de que en estos momentos disminuye la producción de figuras en general, las pocas que se realizan presentan una mayor complejidad y decoración, aunque será en aquellas figuras no embarazadas o dando a luz. Partiendo de estas observaciones, cabe preguntarnos: ¿a qué se debe esta diferencia en la complejidad de las figuras?, ¿por qué es en estos momentos cuando se comienza a representar esta actividad económica? ¿Acaso era necesario un

\footnotetext{
${ }^{7}$ Grandes unidades de habitación consideradas en la mayor parte de investigaciones como "centros de reunión" o lugares en los que se llevaban a cabo actividades político-ideológicas.
} 
aumento de la natalidad en estos momentos? Si hacían falta más individuos, ¿se ejerció algún tipo de presión sobre la capacidad reproductiva de las mujeres?

Lo que es fundamental señalar, y que en la gran mayoría de las publicaciones se obvia o se omite, es que estas figuras en estado de gestación, dando a luz o con la criatura en brazos, nos están otorgando una información muy valiosa, y es la importancia que esta actividad económica tiene para la continuidad de la sociedad y que, por algún motivo, se está representado en estas esculturas. Estamos hablando de la Producción de Sujetos Sociales y la Producción de Mantenimiento.

\section{La Teoría de la Producción de la Vida Social}

Debemos tener presentes los objetivos de los que partimos para hacer Arqueología, y es conocer la realidad y las condiciones materiales en las que se desarrolló la vida de las mujeres y los hombres del pasado. Es por ello, que consideramos primordial estudiar la producción y el trabajo social, conocer cada situación histórica y qué tipo de relaciones sociales se dieron en cada uno de los contextos históricos que estudiamos: si éstos estaban caracterizados por una simetría (con relaciones de reciprocidad) o asimetría (con relaciones de explotación) entre los colectivos sociales y sexuales que conforman la sociedad (Castro y Escoriza 2004-2005).

Para entender mejor este tema, debemos alejarnos de lo que denominamos la Falacia de la Igualdad, cuyo objetivo es establecer la conocida como "igualdad de oportunidades" en la sociedad. Mediante ésta, se llevan a cabo una serie de normas para lograr establecer un equilibrio de igualdad entre todos y todas, cuando en realidad los contextos y la historia de cada uno de los sujetos, sector social o grupo, es diferente (Castro y Escoriza 2004-2005: 134-35).

De este modo, es fundamental distinguir entre igualdad y simetría social, puesto que cuando hablamos de este último término, realmente nos estamos refiriendo a un equilibrio entre los sujetos sociales. Puede ser entendido como relaciones de reciprocidad, las cuales existirán siempre que no se imponga un poder hegemónico y encontremos una 
compensación ajustada entre los sujetos y colectivos sociales y sexuales. Ésta compensación solo se podrá llevar a cabo a través de una serie de principios de derecho desiguales, estableciendo un desequilibrio mediante unas determinadas discriminaciones que consigan restablecer la simetría entre los sujetos sociales (Castro y Escoriza 2005: 136).

Pero, ¿por qué le damos esta importancia al trabajo? Porque es mediante el trabajo como se transforman las condiciones materiales y sociales, como se modifica la realidad y se crea la vida social tanto en objetos como en otros sujetos, con los que establecemos relaciones en prácticas económicas y político-ideológicas. Son las mujeres y los hombres los/as que llevan a cabo un gasto de energía y de tiempo en la producción material y en la producción de sujetos y de mantenimiento, y asegurar así la continuidad de la realidad social. Sin trabajo, no hay sociedad (Castro, Escoriza y Sanahuja 2002).

En el caso de las Figuras de Valdivia, éstas han sido creadas con una intencionalidad, y concretamente, las figuras embarazadas, dando a luz o cuidando de una criatura, también han sido llevadas a cabo con el propósito de representar a las mujeres durante el desarrollo de un trabajo específico y fundamental para la continuidad social.

En lo que a la Producción de Sujetos se refiere, el Objeto y la Fuerza de Trabajo son el cuerpo de las mujeres, lo que implica que, ante la necesidad de un aumento de la productividad, ésta solo pueda llevarse a cabo mediante un sobretrabajo por parte de las mismas. Uno de los mayores obstáculos que ha supuesto que la Producción de Sujetos no haya sido considerada una actividad económica, ha sido la naturalización de ésta, lo cual ha provocado a su vez que se propicie la explotación de las mujeres como productoras de vida. ¿Cómo observamos entonces la explotación en la Producción de Sujetos? A través de una apropiación de las mujeres y sus cuerpos como Objeto y Fuerza de Trabajo por parte del colectivo masculino, o la usurpación de las criaturas (Producto). El modo de poder enfrentarse a esta situación es a través del control de su propio cuerpo y los productos que proporciona (Castro et al 2001: 21).

Respecto a la Producción de Mantenimiento, esta consiste en la conservación y preservación de los sujetos y objetos sociales. Esta actividad, normalmente se ha 
considerado de un bajo nivel social, puesto que no crea objetos nuevos, sino que realmente se trata del objeto de la producción inicial, sin tener en cuenta que esta labor de mantenimiento, es un trabajo fundamental, puesto que, sin ésta, los objetos y los individuos dejarían de ser útiles o prácticos para el resto de la sociedad. Por tanto, debería ser considerada una actividad económica más, de la que además, vemos que realmente se beneficia todo el conjunto de la sociedad. Sin embargo, a pesar de los argumentos anteriormente desarrollados, se han establecido disimetrías en el trabajo de aquellos/as que se dedican a dicha actividad, de manera que se beneficia a su vez a todos/as los/as que se dedican a otro tipo de producciones (Escoriza 2008).

En este caso, y como vemos representado, la Producción de Mantenimiento normalmente es llevado a cabo por las mujeres, vinculadas con el cuidado de los objetos y sujetos sociales. Normalmente, se las ha denominado como "tareas" y han sido consideradas como actividades voluntarias realizadas por las mujeres. Este trabajo se puede realizar sobre niños/as, ancianos/as, herramientas, alimentos, etc. (Escoriza 2008) Así mismo, vemos cómo estas figuras se han realizado con la intención de representar una actividad productiva como es el mantenimiento de las criaturas.

La compensación por este tipo de actividades de mantenimiento la encontraremos en la medida en la que los sujetos sociales participen de lo producido: la producción social conlleva una participación colectiva, mientras que el consumo o uso de aquello que se ha producido, solo beneficiará o recaerá de manera individual. Así, si el trabajo beneficia a un grupo dominante que se aprovecha de la explotación del trabajo de otros colectivos, y este se considera como "beneficio social", estaríamos diciendo que esta apropiación disimétrica del primer grupo es beneficiosa para la sociedad, contribuyendo a estas relaciones de explotación. La clave para conocer si se dieron determinados privilegios materiales, es atendiendo al principio anterior y no tener únicamente en cuenta el beneficio que puede tener una sociedad a través del trabajo (Castro et al 2001). Es por ello, que sería fundamental conocer quién las realizó y si se dieron grupos sociales y sexuales que se pudiesen beneficiar del uso de las figurillas o que no tuvieran acceso a éstas. 
Es necesario entonces, profundizar ahora en el concepto de explotación. Éste lo podemos entender como la distancia entre producción y consumo, la cual se genera mediante la apropiación. Encontraríamos dos grupos sociales en este proceso de explotación: los que usurpan y los que se ven en la situación de ser apropiados (cuerpo, fuerza de trabajo, medios de producción y productos). Esta apropiación, puede verse acompañada de sistemas de alienación: mecanismos para ocultar o alejar de la conciencia colectiva estos sistemas de usurpación (Castro et al 2001: 21). Cabría entonces preguntarnos, ¿fueron empleadas estas figuras como un elemento de alienación para desarrollar algún mecanismo de explotación? Por ejemplo, ¿se realizaron para insistir en la necesidad de un aumento en la producción de sujetos sociales por necesidades demográficas? ¿fue un mecanismo de insistencia para que el mantenimiento de los sujetos recaiga sobre el colectivo femenino?

Partiendo de lo anteriormente expuesto, cabría plantearse un análisis sobre la división sexual del trabajo que vemos representado en las figuras. Como hemos visto, en lo que respecta a la Producción de Sujetos Sociales, la principal diferencia la tenemos en la base biológica de nuestra especie: la capacidad de dar y gestar vida, una especialización del trabajo que se da en todas las sociedades, lo que conlleva que sea necesario ese equilibrio o compensación que lleve consigo una sociedad simétrica (Castro et al 1998). Es por ello por lo que reiteramos sobre la necesidad de sexuar el pasado, tal y como señalamos en la Teoría de las Representaciones Figurativas. Insistimos sobre la importancia de no confundir conceptos como "diferencia" con desigualdad, dominio o explotación. El concepto de diferencia ha sido degradado en manos del Patriarcado para oprimir a las mujeres como colectivo, para establecer una jerarquía entre colectivos sociales y para ejercer el sometimiento, explotación y violencia sobre sus cuerpos y sus vidas. Cuando algo se sale de las "normas excluyentes" se tiende a invisibilizar, por lo que las actividades realizadas por el colectivo femenino tienden a naturalizarse, dejándose de considerar imprescindibles, de manera que la diferencia sexual pasa a ser un indicador de desigualdad, estableciendo sistemas de explotación, discriminación, insolidaridad, exclusión, marginación, etc. (Castro y Escoriza 2005). 
Por todo lo analizado anteriormente, insistimos en la necesidad de realizar análisis bioarqueológicos, para conocer a través de los enterramientos las condiciones de vida de estas mujeres: el número de partos, la salud reproductiva de las mujeres, si se dio algún tipo de violencia de un colectivo sobre otro (por ejemplo a través de la presión reproductiva, tasa de mortalidad de las mujeres, etc.). De este modo, estaremos hablando de explotación sobre el colectivo femenino cuando: la mayor parte del trabajo recae en las mujeres y lo producido beneficia a todo el grupo, cuando los tiempos de trabajo son desequilibrados y extenuantes para el colectivo femenino pero beneficia a los hombres, y cuando lo producido privilegia al colectivo masculino. Una de las principales consecuencias de esta explotación es la negación del trabajo de las mujeres y la invisibilización de la participación de las mismas en la producción de la vida social.

\section{Consideraciones finales}

Por tanto, y tomando como marco de referencia lo anteriormente expuesto, proponemos una nueva perspectiva de análisis de las figuras de Valdivia no sólo como objetos en sí mismos, sino entendidas como materialidad social, producto de un trabajo específico, además de adentrarnos en las prácticas político-ideológicas en las que pudieron verse involucradas (García 2006: 25). Además, es fundamental ponerlas en relación con el contexto arqueológico y en relación con otros objetos.

Es necesario también, estudiar aquellas figuras que han sido realizadas representando las actividades de dar y producir vida, desde un punto de vista de la Teoría de la Producción de la Vida Social, es decir, a través de una perspectiva en la que las mujeres se manifiestan como productoras de sujetos sociales y de mantenimiento, actividades económicas primordiales. Por este motivo, volvemos a insistir en la necesidad de sexuar el pasado, y en este caso, las mismas figuras.

Así mismo, consideramos fundamental conocer cómo se gestionaron las relaciones que se pudieron dar entre sexos, si fueron de reciprocidad o explotación, si hubo mecanismos de violencia de un colectivo sexual sobre otro, si se dio una división 
sexual del trabajo (distinta a la Producción de Sujetos), etc. Además, sería interesante abordar el volumen de trabajo necesario en la producción de sujetos sociales a través de, por ejemplo, la medición de parámetros demográficos y vincularla a las necesidades de esta actividad productiva. Conocer de este modo la existencia o no de periodos en los que se fuese necesario aumentar la productividad en cuanto a producción de sujetos se refiere, el mantenimiento del nivel poblacional, etc. ya que serían indicios de un posible sobretrabajo.

Debemos tener presente, la forma en la que se ha venido tratando la diferencia de las mujeres, normalmente entendida de manera restringida, a través de un modo en el que se proporciona una imagen de las mujeres y de su cuerpo fragmentado (Lagarde 2003). A modo de conclusión, y tal y como señala la Dra. Trinidad Escoriza (2002), este hecho ha llevado consigo el otorgar al colectivo femenino toda una serie de tareas y funciones específicas desde la Prehistoria, dando lugar a una imagen de incapacidad o imposibilidad para la realización de otras tareas debido a su sexo. Es el Patriarcado el que se ha encargado de ocultar y desacreditar la diferencia sexual en todos los ámbitos, por ejemplo, a través de la naturalización de las Producciones de Mantenimiento y de Sujetos Sociales, y de prácticas político-ideológicas, mediante la disposición de los contenidos que se representan y las formas de las representaciones.

\section{Referencias bibliográficas}

Ayala, Enrique y Fernández, Sonia. 1995. Ecuador: las raíces del presente. Quito: Universidad Andina Simón Bolívar.

Barroso, Gonzalo. 2014. "La cultura Valdivia o el surgimiento de la cerámica en América”. Historia Digital,vol. 14: 6-22.

Baumann, Peter. 1985. Valdivia. El descubrimiento de la más antigua cultura de América. Barcelona: Editorial Planeta.

Capua, Constanza di. 2002. De la imagen al icono. Estudios de arqueología e historia del Ecuador. Quito: Abya-Yala. 
Castro, Pedro; Gilí, Silvia; Lull, Vicente; Micó, Rafael; Rihuete, Cristina; Risch, Roberto y Sanahuja, Ma Encarna. 1998. "Teoría de la Producción de la Vida Social. Mecanismos de explotación en el sudeste ibérico". Boletín de antropología americana, $\mathrm{n}^{\mathrm{o}} 33$ : 25-77.

Castro, Pedro; Escoriza, Trinidad; Oltra, Joaquim; Otero, Montserrat y Sanahuja, $\mathbf{M}^{\mathrm{a}}$ Encarna. 2003. "¿Qué es una ciudad? Aportaciones para su definición desde la Prehistoria”. Script Nova 7. Barcelona: Universidad de Barcelona.

Castro, Pedro; Escoriza, Trinidad y Sanahuja, M Encarna. 2003. "Trabajo, reciprocidad y explotación. Prácticas sociales, sujetos sexuados y condiciones materiales". Cultura y Política. IX Congreso de Antropología. Barcelona: Institut Català d’Antropologia.

Castro, Pedro; Escoriza, Trinidad, M $\mathbf{M}^{\mathrm{a}}$ Inés Fregeiro, Joaquim Oltra, Montserrat Otero y Encarna Sanahuja. 2006. Contra la falsificación del pasado prehistórico. Buscando la realidad de las mujeres y los hombres detrás de los estereotipos. Madrid: Instituto de la Mujer.

Castro, Pedro y Trinidad Escoriza. 2004-2005. “Trabajo y sociedad en arqueología. Producciones y relaciones versus orígenes y desigualdades". Revista AtlánticaMediterránea de Prehistoria y Arqueología Social (RAMPAS), nº 7: 131-147.

. 2009. "Lugares reales y lugares ideales. Realidad y construcción de ficciones en Arqueología." Cuadernos de Prehistoria y Arqueología de la Universidad de Granada, no 19: 37-64.

2011. "¿Tal como éramos? Reconstrucciones, ficciones y diseños en la interpretación de las representaciones figurativas de las sociedades ágrafas." Revista Atlántica-Mediterránea de Prehistoria y Arqueología Social, nº 13: 97-118.

Escoriza, Trinidad y Sanahuja, $\mathbf{M}^{\mathrm{a}}$ Encarna. 2002. "Cuerpos de mujeres: Teoría de las representaciones figurativas.”. Congreso Interdisciplinar. Educación y Género.

2002. "El pasado no es neutro: el cuerpo femenino como materialidad y forma de representación social". Las Mujeres en la Historia de Andalucía. 3er Congreso Internacional de Historia de Andalucía. Córdoba: Universidad de Córdoba. 243-258.

Escoriza, Trinidad. 2008. Mujeres y arqueología. Granada: Junta de Andalucía. Consejería de Cultura.

García, Mariella. 2006. Las figurinas de Real Alto: reflejos de los modos de vida Valdivia. Quito: Abya-Yala.

Hill, Betsy. 1975. "A new Chronology of the Valdivia Ceramic Complex from the coastal zone of Guayas Province". Newpa Pacha: Journal of Andean Archaeology, $\mathrm{n}^{\circ} 10$ : 1-32. 
Lathrap, Donald y Jorge G. Marcos. 1975. "Informe preliminar sobre las excavaciones del sitio de Real Alto por la Misión Antropológica de la Universidad de Illinois”. Revista de la Universidad Católica, no 3: 41-46.

Lagarde, Marcela. 2003. Los cautiverios de las mujeres: madresposas, monjas, putas, presas y locas. México D.F: Universidad Nacional Autónoma de México.

López, Erick. 1996. "La Venus Valdivia de Río Chico (OMJPLP-170A): costa sur de la Provincia de Manabí". Boletín Arqueológico, $\mathrm{n}^{\circ}$ 5: 157-174. Guayaquil: Centro de Estudios Arqueológicos y Antropológicos de la ESPOL.

Marcos, Jorge G. 2005. Los pueblos navegantes del Ecuador prehispánico. Quito: AbyaYala.

Marcos, Jorge G. 2012. La historia prehispánica de los pueblos Manteño Huancavilca de Chanduy. Quito: Universidad Internacional del Ecuador.

Sanahuja, Mª Encarna. 2002. Cuerpos sexuados, objetos y prehistoria. Madrid: Cátedra. 